\title{
Efficacy and Safety of Phytosomal Curcumin in Non-Alcoholic Fatty Liver Disease: A Randomized Controlled Trial
}

\author{
Authors \\ Yunes Panahi', Parisa Kianpour ${ }^{2}$, Reza Mohtashami ${ }^{3}$, Ramezan Jafari ${ }^{4}$, \\ Luis E. Simental-Mendía ${ }^{5}$, Amirhossein Sahebkar6,7 \\ Affiliations \\ 1 Chemical Injuries Research Center, Baqiyatallah University of \\ Medical Sciences, Tehran, Iran \\ 2 Pharmaceutical Sciences Branch, Islamic Azad University, \\ Tehran, Iran \\ 3 Religion and Medicine Research Center, Baqiyatallah University of \\ Medical Sciences, Mashhad, Iran \\ 4 Baqiyatallah University of Medical Sciences, Mashhad, Iran \\ 5 Biomedical Research Unit, Mexican Social Security Institute, \\ Durango, Mexico \\ 6 Biotechnology Research Center, Mashhad University of Medical \\ Sciences, Mashhad, Iran \\ 7 Metabolic Research Centre, Royal Perth Hospital, School of \\ Medicine and Pharmacology, University of Western Australia, \\ Perth, Australia
}

Key words

curcuminoids, randomized controlled trial, non-alcoholic fatty liver disease, liver, steatohepatitis

received 05.11.2016

accepted 22.12.2016

\section{Bibliography}

DOI http://dx.doi.org/10.1055/s-0043-100019

Published online: February 3, 2017 | Drug Res 2017; 67: 244-251

(c) Georg Thieme Verlag KG Stuttgart · New York

ISSN 2194-9379

\section{Correspondence}

Amirhossein Sahebkar, PharmD, PhD

Department of Medical Biotechnology

School of Medicine
Mashhad University of Medical Sciences

Mashhad, Iran, P.O. Box: 9177948564

Iran

Tel.: + 98/511/8002 288, Fax: + 98/511/8002 287

sahebkara@mums.ac.ir

amir_saheb2000@yahoo.com

amirhossein.sahebkar@uwa.edu.au

\section{ABSTRACT}

Objective Non-alcoholic fatty liver disease (NAFLD) is a common liver disease characterized by excess lipid deposition in the hepatic tissue and subsequent oxidative and inflammatory damage. Curcumin is a dietary polyphenol with lipid-modifying, antioxidant and anti-inflammatory properties. This study aimed to evaluate the efficacy and safety of supplementation with phytosomal curcumin in subjects with NAFLD. Methods Patients diagnosed with NAFLD (grades 1-3 according to liver ultrasonography) were randomly assigned to the curcumin (phytosomal form; $1000 \mathrm{mg} /$ day in 2 divided doses) $(n=50)$ or placebo group $(n=52)$ for a period of 8 weeks. All patients received dietary and lifestyle advises before the start of trial. Anthropometric measurements, hepatic enzymes, and liver ultrasonography were assessed at baseline and after 8 weeks of follow-up.

Results 87 subjects ( $n=44$ and 43 in the curcumin and control group, respectively) completed the trial. Supplementation with curcumin was associated with a reduction in body mass index $(-0.99 \pm 1.25 \mathrm{vs}$. $-0.15 \pm 1.31$ in the curcumin and placebo groups, respectively; $\mathrm{p}=$ $0.003)$ and waist circumference $(-1.74 \pm 2.58$ vs. $-0.23 \pm 3.49$ in the curcumin and placebo groups, respectively; $p=0.024$ ). Ultrasonographic findings were improved in $75.0 \%$ of subjects in the curcumin group, while the rate of improvement in the control group was $4.7 \%(p<0.001)$. Serum levels of aspartate aminotransferase and alanine aminotransferase were reduced by the end of trial in the curcumin group $(p<0.001)$ but elevated in the control group $(p<0.001)$. Curcumin was safe and well tolerated during the course of trial.

Conclusion Short-term supplementation with curcumin improves liver fat and transaminase levels in patients with NAFLD.

\section{Introduction}

Non-alcoholic fatty liver disease (NAFLD) is a common hepatic disease characterized by fatty infiltration in hepatocytes [1]. The prevalence NAFLD ranges from 15-30\% in the overall Western populations [2-5], while its prevalence increases to 50-90\% in obese individuals, more than $70 \%$ in type 2 diabetic patients and nearly $100 \%$ in those subjects with both conditions [6]. Excessive fat accumulation and insulin resistance appear to play important roles in the pathogenesis of NAFLD [7]. NAFLD encompasses a spectrum of liver abnormalities starting from simple hepatic steatosis, steatohepatitis, liver fibrosis and cirrhosis which can finally progress to hepatocellular carcinoma [8]. Although pathogenesis of NAFLD is complex and remains uncertain, it has been hypothesized that lipid deposition in the liver is the primary event involved and subsequently a variety of secondary processes, such as oxidative stress, lipid peroxidation, inflammatory response, hepatic fibrosis and apoptosis, are triggered [9]. Owing to the increased global rates of obesity, a condition that is strongly associated with NAFLD, development of novel effective therapies with minimal side-effects to control or slow the progression of this disease is required.

Curcumin is a yellow phenolic compound that was initially isolated from Curcuma longa L. (turmeric) rhizomes in 1815 [10]. Curcumin has been shown to possess numerous pharmacological effects including antioxidant, anticarcinogenic, anti-inflammatory, antidepressant, lipid-modifying, anti-arthritic, properties have been described for this polyphenol [11-23]. Numerous pharmaco- 
logical properties of curcumin are due to the interaction of this natural product with various molecular targets such as enzymes, receptors, transcription factors, growth factors, cytokines, adipokines, and other important biomolecules [24-29]. Furthermore, this polyphenol improves insulin sensitivity $[15,30,31]$ and mitochondrial function [32], suppresses adipogenesis [33], reduces inflammation [34, 35], fibrosis [36], and oxidative stress [37-39], that are causal risk factors of NAFLD. More importantly, experimental studies in rodents with diet-induced NAFLD have suggested that curcumin treatment can reduce hepatic lipid accumulation and progression of NAFLD to non-alcoholic steatohepatitis (NASH) [40,41]. In clinical practice, although there is evidence on the efficacy of curcumin supplementation in lowering both plasma triglyceride and cholesterol levels [42-46], there has been no study targeting subjects with NAFLD. Hence, the aim of the present study was to evaluate the efficacy and safety of supplementation with curcumin in subjects with NAFLD.

\section{Materials and Methods}

\section{Subjects}

Studied subjects were selected from adults referring to the Gastroenterology and Hepatology Clinic of the Baqiyatallah Hospital (Tehran, Iran) for whom a diagnosis of NAFLD (grades 1-3) was made according to liver ultrasonography. Exclusion criteria were pregnancy or breastfeeding, NAFLD secondary to alcohol consumption, smoking, consumption of hypoglycemic, hypolipidemic and anti-inflammatory medications as well as any drug known to affect hepatic function, and presence of hepatitis, coronary, renal, pulmonary and thyroid diseases. All patients received dietary and lifestyle advises before the start of trial.

Eligible subjects were randomly (via alternative assignment to capsules bottles coded as A or B) allocated to the curcumin ( $1000 \mathrm{mg} /$ day in 2 divided doses) $(n=50)$ or control $(n=52)$ group. Curcumin was administered in the form of $500 \mathrm{mg}$ capsules, and patients were advised to take the capsules after meal. Administered curcumin had a phytosomal formulation (Meriva ${ }^{\circledR}$; Indena S.p.A, Milan, Italy) that contained a complex of curcumin and soy phosphatidylcholine in a 1:2 weight ratio, and 2 parts of microcrystalline to improve flowability, with an overall content of curcumin in the final product of around $20 \%$ [47].

The study protocol was given approval by the institutional Ethics Committee and written informed consent was obtained from participants. The clinical trial protocol has been registered under the Iranian Registry of Clinical Trials (IRCT) ID: IRCT2015122525641N2.

\section{Anthropometric measurements}

Height and weight of subjects were measured with the accuracy of $0.1 \mathrm{~cm}$ and $0.1 \mathrm{~kg}$, respectively. Measurements were performed in the standing position, with minimal clothing and no shoes at baseline and the $8^{\text {th }}$ week. Body mass index was calculated as weight $(\mathrm{kg})$ divided by height $(\mathrm{m})$ squared.

\section{Blood sampling and biochemical measurements}

Fasted blood samples were collected from the drawn from a cubital vein at baseline and after 8 weeks of supplementation. Blood samples were centrifuged for $10 \mathrm{~min}$ at a speed of $2000-1500 \mathrm{rpm}$ to separate the serum. Serum samples were kept at $-80+{ }^{\circ} \mathrm{C}$ until analyses. Serum levels of alanine aminotransferase (ALT), aspartate aminotransferase (AST), alkaline phosphatase (ALP), total and direct bilirubin were measured at baseline and at the end of study using routine enzymatic assays with commercial kits (Pars Azmoon, Tehran, Iran).

\section{Liver doppler sonography}

Sonography of the liver is performed when the patient has fasted for $8-12 \mathrm{~h}$ before the examination. The examination is performed using 3.5-or 5- $\mathrm{MHz}$ convex probe.

Liver fat content and the severity of hepatic steatosis was evaluated at baseline and after 8 weeks of supplementation using ultrasonography with a Mindray DC-8 diagnostic ultrasound system (convex 3.5-5.0 MHz) at the beginning and the end of the study. Ultrasound assessments were performed by the same expert radiologist blinded to the type of allocation, and with the same instrument. Assessments were performed in the fasted state (8-12 h), with the subjects in the supine position.

Right and left lobes of the upper and lower surfaces of liver were studied. Echogenicity of the liver, the presence or absence of bulky tumors cystic or solid and calcification was assessed. Intrahepatic bile ducts, portal vein and hepatic artery were evaluated. Hepatic steatosis was graded as 0 (lack of fat accumulation), 1 (mild increase in echogenicity with normal visualization of the diaphragm and intrahepatic vessel borders), 2 (moderate increase in echogenicity with slightly impaired visualization of the diaphragm and intrahepatic vessel borders), and 3 (severe increase in echogenicity with markedly impaired visualization of the diaphragm, intrahepatic vessel borders and the posterior portion of the right hepatic lobe).

The portal vein was evaluated in a sagittal oblique view demonstrating the vessels' longest axis. The antegrade hepatopetal flow throughout the entire cardiac cycle was considered as the portal vein flow. The length of the liver on the sagittal view in the mid-clavicular line was considered as liver size and was measured in the left posterior-oblique position.

\section{Statistical analysis}

Statistical analyses were performed using the SPSS software version 11.5 (SPSS Inc., Chicago, Illinois, USA). The study population size was estimated at the significance level of $95 \%$, with a power of $80 \%$ and an effect size of 0.7 for AST and ALT. Data were expressed as mean \pm SD or number (\%). Within-group comparisons were performed using paired samples t-test (for normally distributed data) or Wilcoxon signed-ranks test (for non-normally distributed data). Between-group comparisons were performed using independent samples t-test (for normally distributed data) or Mann-Whitney U test (for non-normally distributed data). Categorical variables were compared using Fisher's Exact test. Binary logistic regression analysis was used to adjust for the effect of potential confounders on the association between curcumin supplementation and changes the severity of NAFLD according to ultrasonographic findings. For this analysis, change in the ultrasonographic findings (categorized as either improvement or lack of improvement) was entered into the model as dependent variable. A p-value of $<0.05$ was considered as statistically significant in all analyses. All analyses were performed per protocol. 


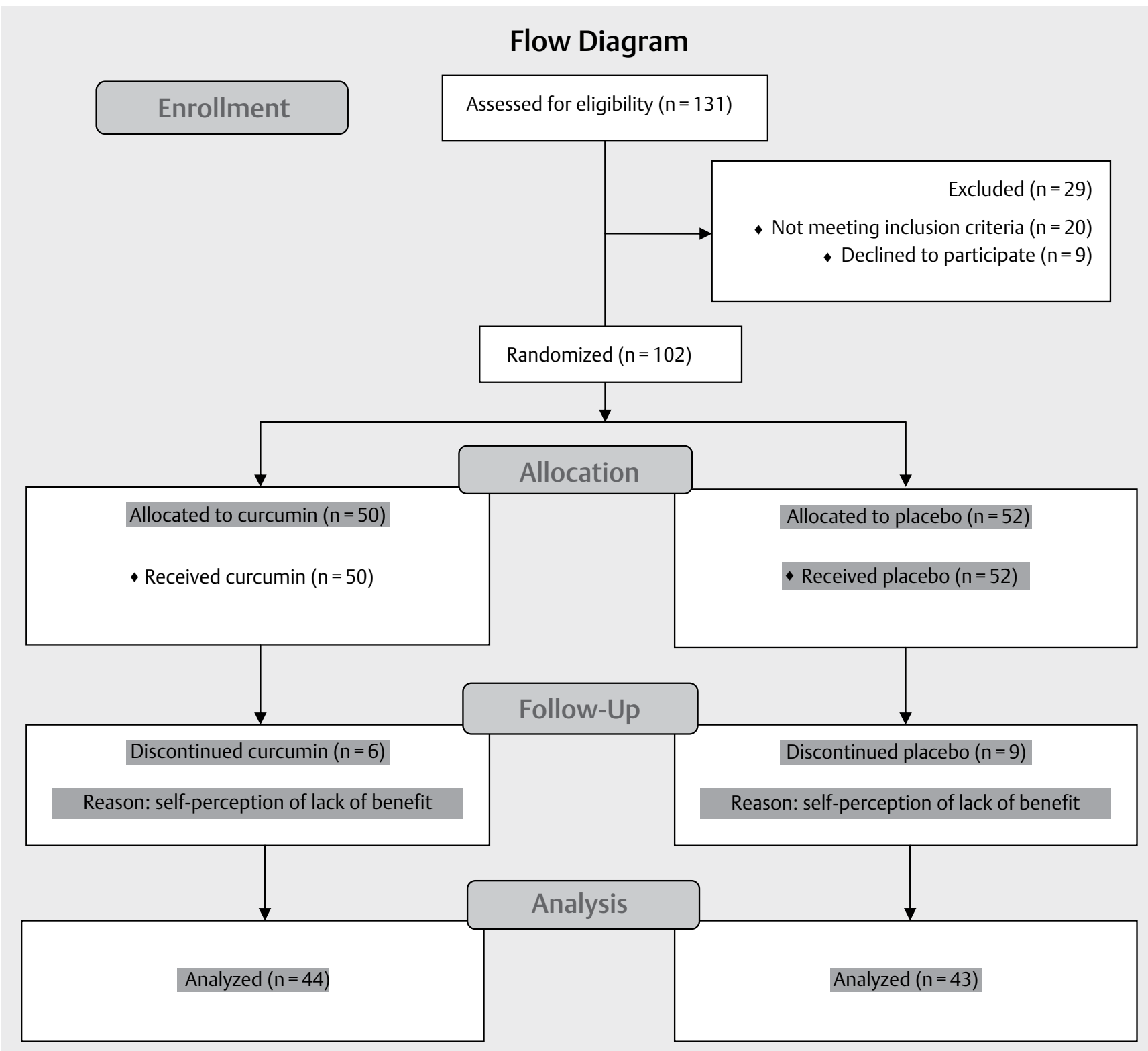

- Fig. 1 Flow diagram of study.

\section{Results}

\section{Baseline characteristics}

86 subjects completed the study. There were 6 drop-outs in the curcumin group and 9 in the placebo group. All drop-outs were because of stopping the medication owing to the self-perception of lack of benefit ( $\triangleright$ Fig. 1). Drop-out rate did not differ between the study groups $(p=0.579)$. The study groups were comparable in terms of age, gender, BMI, SBP, DBP, ALT, AST, ALP, total and direct bilirubin, smoking history and frequency of type 2 diabetes, coronary heart disease, obesity, hypertension, hyperlipidemia and hypertension ( $\triangleright$ Table 1 ). However, there was a significant baseline difference in waist circumference between the study groups ( $\triangleright$ Table 1). The severity of fatty liver based on parenchymal echogenicity and hepatic vein flow were comparable between the groups, yet portal vein diameter and liver volume were different ( Table 1).

\section{Anthropometrics and blood pressure}

Supplementation with curcumin was associated with significant reductions in BMI $(p<0.001)$ and waist circumference $(p<0.001)$ by the end of trial; however, no significant anthropometric change was observed with placebo ( $p>0.05$ ) ( $>$ Table 2$)$. Between-group comparison of changes revealed a significant difference between curcumin and placebo groups with respect to BMI $(p=0.003)$ and waist circumference $(p=0.024)$ ( $\vee$ Table 3$)$. With respect to blood pressure, there was no significant change in SBP and DBP in any of the study group by the end of trial ( $\vee$ Table 2 ), nor was there any significant difference between the groups in terms of blood pressure changes during the course of study ( $p>0.05)$ ( $\triangleright$ Table 3$)$. 
- Table 1 Comparison of baseline and demographic characteristics between the study groups.

\begin{tabular}{|l|c|c|c|}
\hline & Curcumin & Placebo & p-Value \\
\hline N & 44 & 43 & \\
\hline Female (\%) & $20(45.5)$ & $16(37.2)$ & 0.516 \\
\hline Age (y) & $44.98 \pm 12.59$ & $47.21 \pm 10.29$ & 0.369 \\
\hline BMI (kg/m) & $28.97 \pm 3.42$ & $29.07 \pm 3.48$ & 0.899 \\
\hline $\begin{array}{l}\text { Waist } \\
\text { circumference (cm) }\end{array}$ & $97.07 \pm 11.04$ & $101.90 \pm 11.43$ & 0.048 \\
\hline SBP (cmHg) & $12.00 \pm 0.94$ & $12.32 \pm 1.07$ & 0.126 \\
\hline DBP (cmHg) & $7.82 \pm 0.62$ & $8.02 \pm 0.46$ & 0.116 \\
\hline $\begin{array}{l}\text { Total bilirubin (mg) } \\
\text { dL) }\end{array}$ & $0.73 \pm 0.17$ & $0.77 \pm 0.23$ & 0.354 \\
\hline $\begin{array}{l}\text { Direct bilirubin } \\
\text { (mg/dL) }\end{array}$ & $0.42 \pm 0.15$ & $0.36 \pm 0.14$ & 0.075 \\
\hline ALT (U/L) & $35.46 \pm 22.97$ & $36.81 \pm 24.32$ & 0.790 \\
\hline AST (U/L) & $27.63 \pm 11.35$ & $27.44 \pm 10.01$ & 0.934 \\
\hline ALP (U/L) & $172.52 \pm 40.65$ & $170.77 \pm 40.80$ & 0.841 \\
\hline $\begin{array}{l}\text { Hepatic vein flow } \\
\text { velocity (cm/sec) }\end{array}$ & $17.15 \pm 2.16$ & $17.29 \pm 3.44$ & 0.829 \\
\hline $\begin{array}{l}\text { Portal vein } \\
\text { diameter (mm) }\end{array}$ & $11.41 \pm 1.18$ & $10.54 \pm 1.99$ & 0.015 \\
\hline Liver size (mm) & $154.75 \pm 11.62$ & $160.12 \pm 11.89$ & 0.036 \\
\hline Obesity (\%) & $12(27.3)$ & $14(32.6)$ & 0.644 \\
\hline Hypertension (\%) & $9(20.5)$ & $12(27.9)$ & 0.461 \\
\hline Dyslipidemia (\%) & 21 (47.7) & $29(67.4)$ & 0.083 \\
\hline Diabetes (\%) & $11(25.0)$ & $10(23.3)$ & 1.000 \\
\hline CHD (\%) & $6(13.6)$ & $6(14.0)$ & 1.000 \\
\hline $\begin{array}{l}\text { Values are expressed as mean } \pm \text { SD or number } \\
\text { blood pressure; DBP: diastolic blood pressure; ALT: alanine } \\
\text { aminotransferase; AST: aspartate aminotransferase; ALP: alkaline } \\
\text { phosphatase; CHD: coronary heart disease }\end{array}$ & & \\
\hline & & & \\
\hline
\end{tabular}

- Table 2 Within-group comparison of biochemical parameters between curcumin and placebo groups.

\begin{tabular}{|c|c|c|c|c|c|c|}
\hline & \multicolumn{3}{|c|}{ Curcumin } & \multicolumn{3}{|c|}{ Placebo } \\
\hline & Pre-trial & Post-trial & p-Value & Pre-trial & Post-trial & $p$-Value \\
\hline BMI $\left(\mathrm{kg} / \mathrm{m}^{2}\right)$ & $28.97 \pm 3.42$ & $27.98 \pm 3.05$ & $<0.001$ & $29.07 \pm 3.48$ & $28.92 \pm 3.69$ & 0.461 \\
\hline Waist circumference $(\mathrm{cm})$ & $97.07 \pm 11.04$ & $95.33 \pm 10.77$ & $<0.001$ & $101.90 \pm 11.43$ & $101.66 \pm 11.25$ & 0.664 \\
\hline SBP (cmHg) & $12.00 \pm 0.94$ & $12.07 \pm 0.69$ & 0.417 & $12.32 \pm 1.07$ & $12.23 \pm 0.75$ & 0.493 \\
\hline $\mathrm{DBP}(\mathrm{cmHg})$ & $7.82 \pm 0.62$ & $7.89 \pm 0.62$ & 0.439 & $8.02 \pm 0.46$ & $7.91 \pm 0.65$ & 0.251 \\
\hline Total bilirubin (mg/dL) & $0.73 \pm 0.17$ & $0.66 \pm 0.17$ & $<0.001$ & $0.77 \pm 0.23$ & $0.72 \pm 0.18$ & 0.001 \\
\hline Direct bilirubin (mg/dL) & $0.42 \pm 0.15$ & $0.40 \pm 0.14$ & 0.162 & $0.36 \pm 0.14$ & $0.37 \pm 0.14$ & 0.793 \\
\hline $\operatorname{ALT}(\mathrm{U} / \mathrm{L})$ & $35.46 \pm 22.97$ & $24.85 \pm 12.84$ & $<0.001$ & $36.81 \pm 24.32$ & $41.33 \pm 23.97$ & $<0.001$ \\
\hline AST (U/L) & $27.63 \pm 11.35$ & $20.68 \pm 6.65$ & $<0.001$ & $27.44 \pm 10.01$ & $31.23 \pm 12.80$ & $<0.001$ \\
\hline $\operatorname{ALP}(U / L)$ & $172.52 \pm 40.65$ & $172.91 \pm 39.85$ & 0.912 & $170.77 \pm 40.80$ & $172.59 \pm 43.37$ & 0.399 \\
\hline $\begin{array}{l}\text { Hepatic vein flow velocity } \\
(\mathrm{cm} / \mathrm{sec})\end{array}$ & $17.15 \pm 2.16$ & $21.35 \pm 3.35$ & $<0.001$ & $17.29 \pm 3.44$ & $15.04 \pm 3.88$ & $<0.001$ \\
\hline Portal vein diameter $(\mathrm{mm})$ & $11.41 \pm 1.18$ & $10.70 \pm 1.05$ & $<0.001$ & $10.54 \pm 1.99$ & $11.49 \pm 1.61$ & $<0.001$ \\
\hline Liver size (mm) & $154.75 \pm 11.62$ & $152.82 \pm 10.17$ & 0.003 & $160.12 \pm 11.89$ & $161.88 \pm 27.03$ & $<0.001$ \\
\hline
\end{tabular}

\section{Liver doppler solography}

Comparison of liver ultrasonographic findings revealed a significant improvement in the curcumin vs. placebo group $(p<0.001)$ ( $\triangleright$ Table 4). Ultrasonographic findings were improved in $75.0 \%$ of subjects in the curcumin group, while the rate of improvement in the placebo group was $4.7 \%(p<0.001)$. The frequency of increased liver fat content in the curcumin group was $4.5 \%$ but $25.6 \%$ of subjects in the placebo group had their liver fat content increased $(p=0.007)$ ( Table 4).

Findings of Doppler solography indicated an increase in hepatic vein flow $(p<0.001)$ accompanied by reduction of portal vein diameter $(p<0.001)$ and liver volume $(p=0.001)$ following treatment with curcumin, yet reverse effects were observed in the placebo group ( $>$ Table 2 ). Between-group comparisons revealed significant improvements in hepatic vein flow $(p<0.001)$, portal vein diameter $(p<0.001)$ and liver volume $(p<0.001)$ in the curcumin vs. placebo group ( $\triangleright$ Table 3 ).

\section{Liver enzymes and bilirubin}

Consistent with the findings of liver ultrasonography, serum levels of AST and ALT were reduced by the end of trial in the curcumin group $(p<0.001)$ but elevated in the placebo group $(p<0.001)$ ( $\triangleright$ Table 2$)$. The effect of curcumin in reducing serum AST $(p<0.001)$ and ALT $(p<0.001)$ levels was also significant in the between-group comparison ( $\vee$ Table 3). Comparison of baseline vs. end-trial serum ALP concentrations did not reveal a significant alteration in any of the study groups ( $\triangleright$ Table 2 ), nor was there a significant difference between the groups in terms of ALP changes during the study pe$\operatorname{riod}(p>0.05)(\vee$ Table 3$)$. With respect to total and direct bilirubin concentrations, no significant difference was observed between curcumin and placebo groups $(p>0.05)(\triangleright$ Table 2,3$)$.

\section{Regression analysis}

Binary logistic regression analysis was performed to adjust the ultrasonographic findings - as the primary efficacy measure - for baseline waist circumference, liver volume, portal vein diameter, 
presence of dyslipidemia and serum direct bilirubin concentrations as potential confounders of treatment response. Selection of confounders was based on the baseline difference between curcumin and placebo groups using a p-value cut-off of 0.1 . The effect of curcumin in reducing liver fat remained significant $(p<0.001)$ after adjustment for the above-mentioned confounders ( $\vee$ Table 5 ). The crude unadjusted effect of curcumin supplementation on the improvement of liver fat content was 61.50 (95\% Cl: 12.73, 297.04) $(\mathrm{p}<0.001)$.

\section{Safety}

Curcumin was safe and well tolerated in this trial. There was no report of severe adverse events.

\section{Discussion}

Findings of the present study suggest that supplementation with phytosomal curcumin is well tolerated and significantly reduces fatty liver parameters in individuals with NAFLD. This is consistent with the results of previous studies showing that curcumin regu-

- Table 3 Between-group comparison of biochemical parameters between curcumin and placebo groups.

\begin{tabular}{|l|c|c|r|}
\hline & Curcumin & Placebo & $p$-Value \\
\hline BMI (kg/m $\mathbf{m}^{\mathbf{2}}$ & $-0.99 \pm 1.25$ & $-0.15 \pm 1.31$ & 0.003 \\
\hline $\begin{array}{l}\text { Waist circumfer- } \\
\text { ence (cm) }\end{array}$ & $-1.74 \pm 2.58$ & $-0.23 \pm 3.49$ & 0.024 \\
\hline SBP (cmHg) & $0.07 \pm 0.59$ & $-0.08 \pm 0.96$ & 0.185 \\
\hline DBP (cmHg) & $0.07 \pm 0.59$ & $-0.12 \pm 0.66$ & 0.116 \\
\hline $\begin{array}{l}\text { Total bilirubin } \\
\text { (mg/dL) }\end{array}$ & $-0.07 \pm 0.08$ & $-0.06 \pm 0.11$ & 0.271 \\
\hline $\begin{array}{l}\text { Direct bilirubin } \\
\text { (mg/dL) }\end{array}$ & $-0.02 \pm 0.10$ & $0.005 \pm 0.12$ & 0.271 \\
\hline ALT (U/L) & $-10.61 \pm 15.49$ & $4.51 \pm 7.40$ & $<0.001$ \\
\hline AST (U/L) & $-6.95 \pm 7.47$ & $3.79 \pm 6.43$ & $<0.001$ \\
\hline ALP (U/L) & $0.39 \pm 22.96$ & $1.82 \pm 14.00$ & 0.727 \\
\hline $\begin{array}{l}\text { Hepatic vein flow } \\
\text { velocity (cm/sec) }\end{array}$ & $4.20 \pm 2.77$ & $-2.25 \pm 2.78$ & $<0.001$ \\
\hline $\begin{array}{l}\text { Portal vein } \\
\text { diameter (mm) }\end{array}$ & $-0.70 \pm 0.73$ & $0.95 \pm 1.43$ & $<0.001$ \\
\hline Liver size (mm) & $-1.93 \pm 4.00$ & $1.76 \pm 22.89$ & $<0.001$ \\
\hline
\end{tabular}

Values are expressed as mean \pm SD. SBP: systolic blood pressure; DBP: diastolic blood pressure; ALT: alanine aminotransferase; AST: aspartate aminotransferase; ALP: alkaline phosphatase lates hepatic lipogenesis through AMP-activated protein kinase activation which leads to inhibition of hepatic lipid accumulation [48]. Thus, curcumin can blunt the "first hit" of NAFLD pathogenesis which involves development of hepatic steatosis [9]. Taking both ultrasonographic and biochemical (serum transaminase changes) findings together, it appears that the efficacy of curcumin supplementation in patients with NAFLD is not only statistically significant but also clinically relevant owing to the high improvement rate (75.0\%) that was observed. However, a more definite judgment on the clinical relevance of the effects necessitates longitudinal designs assessing the efficacy of curcumin in preventing or slowing the progression of NAFLD into severe outcomes such as NASH and cirrhosis.

Several studies have indicated potential benefits of curcumin in the prevention and treatment of obesity, diabetes, and metabolic syndrome [49]. Curcumin decreases the production of reactive oxygen species by inhibition of hepatic stellate cell activation and reduction of tissue inhibitor of metalloprotease- 1 release [50]. Another possible mechanism of action of curcumin may involve peroxisome proliferator-activated receptor- $\gamma$ (PPAR- $\gamma$ ) activation which can improve insulin sensitivity [51]. Moreover, the potent antioxidant effect of curcumin in lowering lipid peroxidation and oxidative stress in different tissues including the liver has been previously described [52]. These antioxidant effects of curcumin may be explained by its metal chelating ability modulatory effect on the expression of antioxidant enzymes such as superoxide dismutase, catalase and glutathione peroxidase [53, 54].

Different histological changes may occur during NAFLD including macrovesicular steatosis, lobular inflammatory infiltration, hepatocellular ballooning, formation of Mallory bodies, and perisinusoidal fibrosis [55-58]. It has been demonstrated that curcumin supplementation reduces aminotransferases levels and improves both steatosis and inflammation in liver tissues [59]. Moreover, curcumin inhibits NF-kB activation, decreases downstream induction of ICAM-1, COX-2 and MCP-1, and reduces intrahepatic gene expression of monocyte chemoattractant protein-1, CD11b, procollagen type I, and tissue inhibitor of metalloprotease- 1 leading to the mitigation of the development and progression of hepatic inflammation and fibrosis $[50,60]$. Also, curcumin significantly decreases serum levels of TNF-alpha and IL-6 [61], 2 pro-inflammatory cytokines involved in the "second hit" of the pathogenesis of NAFLD [9]. Lifestyle modification through adherence to a healthy diet and optimal physical activity is the first step in the prevention and treatment of NAFLD $[62,63]$. In the present study, all patients received dietary and lifestyle advises. Along with the observed im-

- Table 4 Comparison of NAFLD severity within and between the study groups.

\begin{tabular}{|c|c|c|c|c|c|c|c|c|}
\hline & & \multicolumn{3}{|c|}{ Curcuminoids } & \multicolumn{3}{|c|}{ Placebo } & \multirow[t]{2}{*}{$p$-Value * } \\
\hline & & Before & After & $\mathbf{p}$ & Before & After & $\mathbf{P}$ & \\
\hline \multirow{4}{*}{ NAFLD severity } & Grade 0 & $0(0)$ & $15(34.1)$ & \multirow{4}{*}{$<0.001$} & $0(0)$ & $1(2.3)$ & \multirow{4}{*}{0.013} & \multirow{4}{*}{$<0.001$} \\
\hline & Grade 1 & 17 (38.6) & $21(47.7)$ & & $17(39.5)$ & $10(23.3)$ & & \\
\hline & Grade 2 & $23(52.3)$ & $6(13.6)$ & & $19(44.2)$ & $21(48.8)$ & & \\
\hline & Grade 3 & $4(9.1)$ & $2(4.5)$ & & $7(16.3)$ & $11(25.6)$ & & \\
\hline
\end{tabular}


Table 5 Binary logistic regression analysis for the effect of curcumin supplementation on liver fat content after adjustment for potential confounders.

\begin{tabular}{|l|c|c|c|}
\hline $\begin{array}{l}\text { Variables entered in } \\
\text { the model }\end{array}$ & $\begin{array}{c}\text { Odds } \\
\text { ratio }\end{array}$ & $\mathbf{9 5 \% ~ C l}$ & $\boldsymbol{p}$-Value \\
\hline $\begin{array}{l}\text { Baseline waist } \\
\text { circumference }\end{array}$ & 1.02 & $0.96,1.09$ & 0.432 \\
\hline Baseline liver size & 0.98 & $0.92,1.03$ & 0.420 \\
\hline $\begin{array}{l}\text { Baseline portal vein } \\
\text { diameter }\end{array}$ & 0.78 & $0.52,1.18$ & 0.237 \\
\hline Curcumin treatment & 96.50 & $14.30,651.22$ & $<0.001$ \\
\hline $\begin{array}{l}\text { Change in the ultrasonographic findings (categorized as either } \\
\text { improvement or lack of improvement) was entered into the model } \\
\text { as dependent variable, and the variables listed in the table were } \\
\text { entered as independent factors }\end{array}$ \\
\hline
\end{tabular}

provements in the ultrasonographic and biochemical indices, curcumin supplementation resulted in a significant reduction in anthropometric indices including BMI and waist circumference. It is worth noting that curcumin has been shown to enhance physical performance and reduce physiological fatique and [64] this might have contributed to the therapeutic effects of curcumin in reducing BMI and other indices of NAFLD.

On the other hand, our results support the efficacy and safety of curcumin supplementation as it has been previously reported by several studies in both healthy subjects and patients with different diseases [65-68].

The main limitation of the present study was that histological changes were not assessed because liver biopsy is an invasive diagnostic test limited by ethical issues; therefore, hepatic findings were evaluated by hepatic ultrasound which has been shown to have high sensitivity, specificity and accuracy for the assessment of NAFLD $[69,70]$. Besides, some other indices such as gamma-glutamyltransferase and spleen size that could serve as indices of NAFLD progression were not assesse din this study and deserve to be measured in future trials of curcumin. Another limitation is the short duration of follow-up which impedes judgment on the long-term efficacy of curcumin supplementation particularly in terms of reducing vascular and hepatic outcomes. Nonetheless, that curcumin exerts protective effects as early as 8 weeks of supplementation is a proof for the relevance of supplementation with this agent. Finally, given the small population size, some between-group differences in baseline characteristics were present in spite of randomization. Hence, confirmatory evidence from intention-to-treat analyses in larger trials would be ideal to confirm the positive findings observed in the present study.

In conclusion, results of this study revealed that short-term curcumin supplementation improves sonographic findings of NAFLD and hepatic transaminase levels. However, future studies are warranted to elucidate if the anti-steatotic properties of curcumin are dose-dependent, and also the value of curcumin in reducing cardiovascular and hepatic endpoints such as cirrhosis and hepatocellular carcinoma.

\section{Acknowledgment}

The authors are grateful for the supports provided by the Baqiyatallah University of Medical Sciences (Tehran, Iran) and Indena SpA (Milan, Italy).

\section{Conflict of Interest}

The authors have no competing interest to declare.

\section{References}

[1] Williamson RM, Price JF, Glancy S et al. Prevalence of and risk factors for hepatic steatosis and nonalcoholic Fatty liver disease in people with type 2 diabetes: the Edinburgh Type 2 Diabetes Study. Diabetes Care 2011; 34: 1139-1144 doi:10.2337/dc10-2229

[2] Clark JM, Diehl AM. Hepatic steatosis and type 2 diabetes mellitus. Curr Diab Rep 2002; 2: 210-215

[3] Bedogni G, Miglioli L, Masutti F et al. Prevalence of and risk factors for nonalcoholic fatty liver disease: the Dionysos nutrition and liver study. Hepatology 2005; 42: 44-52

[4] Browning JD, Szczepaniak LS, Dobbins R et al. Prevalence of hepatic steatosis in an urban population in the United States: impact of ethnicity. Hepatology 2004; 40: 1387-1395

[5] Vernon G, Baranova A, Younossi ZM. Systematic review: the epidemiology and natural history of non-alcoholic fatty liver disease and non-alcoholic steatohepatitis in adults. Aliment Pharmacol Ther 2011; 34: 274-285 doi:10.1111/j.1365-2036.2011.04724.x

[6] Solga SF, Diehl AM. Gut flora-based therapy in liver disease? The liver cares about the gut. Hepatology 2004; 39: 1197-1200

[7] Farrell GC, Larter CZ. Nonalcoholic fatty liver disease: from steatosis to cirrhosis. Hepatology 2006; 43: S99-S112

[8] Adams LA, Lymp JF St, Sauver J et al. The natural history of nonalcoholic fatty liver disease: a population-based cohort study. Gastroenterology 2005; 129: 113-121

[9] Day CP, James OF. Steatohepatitis: a tale of two "hits"? Gastroenterology 1998; 114: 842-845

[10] Gupta SC, Kismali G, Aggarwal BB. Curcumin, a component of turmeric: from farm to pharmacy. Biofactors 2013; 39: 2-13 doi:10.1002/biof.1079

[11] Oetari S, Sudibyo M, Commandeur JN et al. Effects of curcumin on cytochrome P450 and glutathione S-transferase activities in rat liver. Biochem Pharmacol 1996; 51: 39-45

[12] Huang MT, Lysz T, Ferraro T et al. Inhibitory effects of curcumin on in vitro lipoxygenase and cyclooxygenase activities in mouse epidermis. Cancer Res 1991; 51: 813-819

[13] Rao CV, Rivenson A, Simi B et al. Chemoprevention of colon carcinogenesis by dietary curcumin, a naturally occurring plant phenolic compound. Cancer Res 1995; 55: 259-266

[14] Panahi Y, Ghanei M, Bashiri S et al. Short-term curcuminoid supplementation for chronic pulmonary complications due to sulfur mustard intoxication: positive results of a randomized double-blind placebo-controlled Trial. Drug Res (Stuttg) 2015; 65: 567-573 doi:10.1055/s-0034-1389986

[15] Sahebkar A. Why it is necessary to translate curcumin into clinical practice for the prevention and treatment of metabolic syndrome? Biofactors 2013; 39: 197-208 doi:10.1002/biof.1062 
[16] Panahi Y, Hosseini MS, Khalili N et al. Antioxidant and anti-inflammatory effects of curcuminoid-piperine combination in subjects with metabolic syndrome: A randomized controlled trial and an updated meta-analysis. Clin Nutr 2015; 34: 1101-1108 doi:10.1016/j. clnu.2014.12.019

[17] Panahi Y, Saadat A, Beiraghdar F et al. Adjuvant therapy with bioavailability-boosted curcuminoids suppresses systemic inflammation and improves quality of life in patients with solid tumors: a randomized double-blind placebo-controlled trial. Phytother Res 2014; 28: 1461-1467 doi:10.1002/ptr.5149

[18] Panahi Y, Sahebkar A, Parvin S et al. A randomized controlled trial on the anti-inflammatory effects of curcumin in patients with chronic sulphur mustard-induced cutaneous complications. Ann Clin Biochem 2012; 49: 580-588 doi:10.1258/acb.2012.012040

[19] Panahi Y, Rahimnia AR, Sharafi M et al. Curcuminoid treatment for knee osteoarthritis: a randomized double-blind placebo-controlled trial. Phytother Res 2014; 28: 1625-1631 doi:10.1002/ptr.5174

[20] Esmaily $H$, Sahebkar A, Iranshahi M et al. An investigation of the effects of curcumin on anxiety and depression in obese individuals: A randomized controlled trial. Chin J Integr Med 2015; 21: 332-338 doi:10.1007/s11655-015-2160-z

[21] Panahi Y, Badeli R, Karami GR et al. Investigation of the efficacy of adjunctive therapy with bioavailability-boosted curcuminoids in major depressive disorder. Phytother Res 2015; 29: 17-21 doi:10.1002/ ptr.5211

[22] Zabihi NA, Pirro M, Johnston TP et al. Is there a role for curcumin supplementation in the treatment of non-alcoholic fatty liver disease? The data suggest yes. Curr Pharm Des 2016 [Epub ahead of print] PubMed PMID:27748192

[23] Khonche A, Biglarian O, Panahi Y et al. Adjunctive therapy with curcumin for peptic ulcer: a randomized controlled trial. Drug Res (Stuttg) 2016; 66: 444-448

[24] Shehzad A, Lee YS. Curcumin multiple molecular targets mediate multiple pharmacological actions - A review. Drugs Future 2010; 35 : 113-119

[25] Ghandadi M, Sahebkar A. Curcumin: An effective inhibitor of interleukin-6. Curr Pharm Des 2016 [Epub ahead of print] PubMed PMID: 27719643

[26] Karimian MS, Pirro M, Majeed M et al. Curcumin as a natural regulator of monocyte chemoattractant protein-1. Cytokine Growth Factor Rev. 2016, pii: S1359-6101(16)30131-9 doi:10.1016/j.cytogfr.2016.10.001

[27] Panahi Y, Hosseini MS, Khalili N et al. Effects of curcumin on serum cytokine concentrations in subjects with metabolic syndrome: A post-hoc analysis of a randomized controlled trial. Biomed Pharmacother 2016; 82: 578-582 doi:10.1016/j.biopha.2016.05.037

[28] Sahebkar A, Cicero AF, Simental-Mendía LE et al. Curcumin downregulates human tumor necrosis factor- $\alpha$ levels: A systematic review and meta-analysis of randomized controlled trials. Pharmacol Res 2016; 107: 234-242 doi: doi:10.1016/j.phrs.2016.03.026 Review

[29] Momtazi AA, Shahabipour F, Khatibi S et al. Curcumin as a MicroRNA regulator in cancer: a review. Rev Physiol Biochem Pharmacol 2016; 171: 1-38

[30] Na LX, Li Y, Pan HZ et al. Curcuminoids exert glucose-lowering effect in type 2 diabetes by decreasing serum free fatty acids: a double-blind, placebo-controlled trial. Mol Nutr Food Res 2013; 57: 1569-1577 doi:10.1002/mnfr.201200131

[31] Chuengsamarn S, Rattanamongkolgul S, Luechapudiporn R et al. Curcumin extract for prevention of type 2 diabetes. Diabetes Care 2012; 35: 2121-2127 doi:10.2337/dc12-0116

[32] Ramirez-Tortosa MC, Ramirez-Tortosa CL, Mesa MD et al. Curcumin ameliorates rabbits's steatohepatitis via respiratory chain, oxidative stress, and TNF-alpha. Free Radic Biol Med 2009; 47: 924-931
[33] Bradford PG. Curcumin and obesity. Biofactors 2013; 39: 78-87 doi:10.1002/biof.1074

[34] Panahi Y, Sahebkar A, Amiri M et al. Improvement of sulphur mustard-induced chronic pruritus, quality of life and antioxidant status by curcumin: results of a randomised, double-blind, placebo-controlled trial. Br J Nutr 2012; 108: 1272-1279

[35] Sahebkar A. Are curcuminoids effective C-reactive protein-lowering agents in clinical practice? Evidence from a meta-analysis. Phytother Res 2014; 28: 633-642 doi:10.1002/ptr.5045

[36] Kang HC, Nan JX, Park PH et al. Curcumin inhibits collagen synthesis and hepatic stellate cell activation in-vivo and in-vitro. Journal of Pharmacy and Pharmacology 2002; 54: 119-126

[37] Sahebkar A, Mohammadi A, Atabati A et al. Curcuminoids modulate pro-oxidant-antioxidant balance but not the immune response to heat shock protein 27 and oxidized LDL in obese individuals. Phytother Res 2013; 27: 1883-1888 doi:10.1002/ptr.4952

[38] Panahi Y, Alishiri GH, Parvin S et al. Mitigation of systemic oxidative stress by curcuminoids in osteoarthritis: results of a randomized Controlled Trial. J Diet Suppl 2016; 13: 209-220 doi:10.3109/1939021 1.2015.1008611

[39] Panahi Y, Ghanei M, Hajhashemi A et al. Effects of curcuminoids-piperine combination on systemic oxidative stress, clinical symptoms and quality of life in subjects with chronic pulmonary complications due to sulfur mustard: A randomized controlled trial. J Diet Suppl 2016; 13: 93-105 doi:10.3109/19390211.2014.952865

[40] Hasan ST, Zingg JM, Kwan P et al. Curcumin modulation of high fat diet-induced atherosclerosis and steatohepatosis in LDL receptor deficient mice. Atherosclerosis 2014; 232: 40-51

[41] Öner-lyidoğan $Y$, Koçak H, Seyidhanoğlu M et al. Curcumin prevents liver fat accumulation and serum fetuin-A increase in rats fed a high-fat diet. J Physiol Biochem 2013; 69: 677-686

[42] Mohammadi A, Sahebkar A, Iranshahi M et al. Effects of supplementation with curcuminoids on dyslipidemia in obese patients: a randomized crossover trial. Phytother Res 2013; 27: 374-379 doi:10.1002/ptr.4715

[43] Sahebkar A. Curcuminoids for the management of hypertriglyceridaemia. Nat Rev Cardiol 2014; 11: 123 doi:10.1038/nrcardio.2013.140-c1

[44] Sahebkar A. Low-density lipoprotein is a potential target for curcumin: novel mechanistic insights. Basic \& Clinical Pharmacology \& Toxicology 2014; 114: 437-438 doi:10.1111/bcpt. 12212

[45] Panahi Y, Khalili N, Hosseini MS et al. Lipid-modifying effects of adjunctive therapy with curcuminoids-piperine combination in patients with metabolic syndrome: results of a randomized controlled trial. Complement Ther Med 2014; 22: 851-857 doi:10.1016/j. ctim.2014.07.006

[46] Sahebkar A, Chew GT, Watts GF. Recent advances in pharmacotherapy for hypertriglyceridemia. Prog Lipid Res 2014; 56: 47-66 doi:10.1016/j.plipres.2014.07.002

[47] Semalty A, Semalty M, Rawat MS et al. Supramolecular phospholipids-polyphenolics interactions: the PHYTOSOME strategy to improve the bioavailability of phytochemicals. Fitoterapia 2010; 81: 306-314 doi:10.1016/j.fitote.2009.11.001

[48] Um MY, Hwang KH, Ahn J et al. Curcumin attenuates diet-induced hepatic steatosis by activating AMP-activated protein kinase. Basic Clin Pharmacol Toxicol 2013; 113: 152-157 doi:10.1111/bcpt.12076

[49] Aggarwal BB. Targeting inflammation-induced obesity and metabolic diseases by curcumin and other nutraceuticals. Annu Rev Nutr 2010; 30: 173-199 doi:10.1146/annurev.nutr.012809.104755

[50] Vizzutti F, Provenzano A, Galastri S et al. Curcumin limits the fibrogenic evolution of experimental steatohepatitis. Lab Invest 2010; 90: 104-115 doi:10.1038/labinvest.2009.112 
[51] Tang Y, Zheng S, Chen A. Curcumin eliminates leptin's effects on hepatic stellate cell activation via interrupting leptin signaling. Endocrinology 2009; 150: 3011-3020 doi:10.1210/en.2008-1601

[52] Nanji AA, Jokelainen K, Rahemtulla A et al. Activation of nuclear factor kappa $B$ and cytokine imbalance in experimental alcoholic liver disease in the rat. Hepatology 1999; 30: 934-943

[53] Nabavi SF, Eslami S, Moghaddam AH et al. Protective effects of curcumin against fluoride-induced oxidative stress in the rat brain. Neurophysiology 2011; 43: 287-291

[54] Nabavi SF, Nabavi SM, Abolhasani F et al. Cytoprotective effects of curcumin on sodium fluoride-induced intoxication in rat erythrocytes. Bull Environ Contam Toxicol 2012; 88: 486-490 doi:10.1007| s00128-011-0495-5

[55] Tiniakos DG, Vos MB, Brunt EM. Nonalcoholic fatty liver disease: pathology and pathogenesis. Annu Rev Pathol 2010; 5: 145-171 doi:10.1146/annurev-pathol-121808-102132

[56] Brunt EM. Pathology of nonalcoholic fatty liver disease. Nat Rev Gastroenterol Hepatol 2010; 7: 195-203 doi:10.1038/nrgastro.2010.21

[57] Brunt EM. Histological assessment of nonalcoholic fatty liver disease in adults and children. Clin Liver Dis 2012; 1: 107-110

[58] Puri P, Sanyal AJ. Nonalcoholic fatty liver disease: definitions, risk factors, and workup. Clin Liver Dis 2012; 1: 98-102

[59] Ramirez-Tortosa MC, Ramirez-Tortosa CL, Mesa MD et al. Curcumin ameliorates rabbits's steatohepatitis via respiratory chain, oxidative stress, and TNF-alpha. Free Radic Biol Med 2009; 47: 924-931 doi:10.1016/j.freeradbiomed.2009.06.015

[60] Leclercq IA, Farrell GC, Sempoux C et al. Curcumin inhibits NF-kappaB activation and reduces the severity of experimental steatohepatitis in mice. J Hepatol 2004; 41: 926-934
[61] Wang Y, Li J, Zhuge L et al. Comparison between the efficacies of curcumin and puerarin in C57BL/6 mice with steatohepatitis induced by a methionine- and choline-deficient diet. Exp Ther Med 2014; 7 : 663-668

[62] Nseir W, Hellou E, Assy N. Role of diet and lifestyle changes in nonalcoholic fatty liver disease. World J Gastroenterol 2014; 20 : 9338-9344

[63] Finelli C, Tarantino G. Have guidelines addressing physical activity been established in nonalcoholic fatty liver disease? World J Gastroenterol 2012; 18: 6790-6800 doi:10.3748/wjg.v18.i46.6790

[64] Huang WC, Chiu WC, Chuang HL et al. Effect of curcumin supplementation of physiological fatigue and physical performance in mice. Nutrients 2015; 7: 905-921

[65] Nabavi SF, Daglia M, Moghaddam AH et al. Curcumin and liver disease: from chemistry to medicine. Comprehensive Reviews in Food Science and Food Safety. 2014; 13: 62-77 doi:10.1111/1541-4337.12047

[66] Goel A, Kunnumakkara AB, Aggarwal BB. Curcumin as "Curecumin": from kitchen to clinic. Biochem Pharmacol 2008; 75: 787-809

[67] Hatcher H, Planalp R, Cho J et al. Curcumin: from ancient medicine to current clinical trials. Cell Mol Life Sci 2008; 65: 1631-1652 doi:10.1007/s00018-008-7452-4

[68] Soni KB, Kuttan R. Effect of oral curcumin administration on serum peroxides and cholesterol levels in human volunteers. Indian J Physiol Pharmacol 1992; 36: 273-275

[69] Osawa H, Mori Y. Sonographic diagnosis of fatty liver using a histogram technique that compares liver and renal cortical echo amplitudes. J Clin Ultrasound 1996; 24: 25-29

[70] Hernaez R, Lazo M, Bonekamp S et al. Diagnostic accuracy and reliability of ultrasonography for the detection of fatty liver: a meta-analysis. Hepatology 2011; 54: 1082-1090 doi:10.1002/ hep. 24452 